\title{
MEASUREMENT ACCURACY ON 3D POINT CLOUD GENERATED USING MULTISPECTRAL IMAGERY BY DIFFERENT CALIBRATION METHODS
}

\author{
K. Zainuddin ${ }^{1,2} *$ Z. Majid², M. F. M. Ariff ${ }^{2}$, K. M. Idris ${ }^{2}$ \\ ${ }^{1}$ Centre of Study for Surveying Science \& Geomatics, Faculty of Architecture, Planning \& Surveying Universiti Teknologi MARA, \\ Malaysia - khairul760@uitm.edu.my \\ ${ }^{2}$ Geospatial Imaging and Information Research Group, Universiti Teknologi Malaysia, Malaysia. \\ (zulkeplimajid, mfaridma,khairulnizami) @utm.my
}

KEY WORDS: Measurement Accuracy, Calibration, Multispectral Camera, Close-range Photogrammetry, SfM.

\begin{abstract}
:
The state-of-the-art lightweight multispectral cameras are widely used for low altitude remote sensing, also can be exploited as a tool for close-range photogrammetry application. The acquired imagery can be used for generating the 3D model using Structure-fromMotion/ Multi-view Stereo (SfM/MVS) processing software. In photogrammetry, camera calibration is an essential step for accurate measurement. The parameter of the camera system can be estimated using photogrammetric self-calibration bundle-adjustment, or by automatic and straightforward calibration procedure developed by computer vision (CV) community. When using SfM/MVS photogrammetry software, the pre-calibration value is not required, as the algorithm calculates the parameter as a part of point cloud construction process. Nevertheless, processing with the uncalibrated image is only suitable when no metric accuracy required in the modelling project. This paper aims to evaluate the measurement accuracy on generated 3D point cloud based on different estimated parameter method. The evaluation of measurement accuracy started by estimates the camera's interior parameter using two different approaches; photogrammetric self-calibration bundle-adjustment and computer vision calibration. The estimated parameter from both methods then imported into commercial SfM/MVS software to construct the 3D point cloud. The point cloud also generated using uncalibrated images and used for measurement accuracy assessment. All parameters applied to the same datasets involved three different check-fields. Two accuracy assessments were performed by comparing the check-points and check-distance extracted with the total station measurement. As a result, the point cloud generated using photogrammetric approach provides the most accurate result on both assessments. While the automatic on-the-job self-calibration shows inconsistent results.
\end{abstract}

\section{INTRODUCTION}

The multispectral analysis is a branch of remote sensing application, acquiring the imagery at a different wavelength, covering a wide range of spectrum including visible and invisible bands. The multispectral imagery traditionally acquiring from satellite, nowadays can be captured at low altitude using a state-of-the-art lightweight multispectral camera which mounts on Unmanned Aerial Vehicle (UAV). The camera is small in size and low in weight, thus, it can be exploited to be used as a close-range photogrammetry tool for 3D modelling application such as heritage documentation (Torres-Martínez et al., 2017). Using multispectral imagery for 3D modelling provides a low-cost solution for data acquisition and processing by using a single sensor when both geometric and spectrometric analysis documentations are required (Liang et al., 2014; Torres-Martínez et al., 2017).

In Photogrammetry, it is necessary to calibrate the camera to determine the camera's parameters for accurate 3D modelling. Camera calibration is to be known as an essential task to maximise the measurement accuracy of the generated model via self-calibrating bundle adjustment (Remondino and Fraser, 2006; Fraser, 2013; Luhmann et al., 2016). In the other hand, Computer Vision (CV) community also actively developing camera calibration procedure, but more focusing on easy-to-use and fully automatic approach by using a simple 2D flat checkerboard type object as the test-field (Tsai, 1986). Besides, the interior parameter of the camera also can be estimated during point cloud construction on Structure-from-Motion (SfM) processing. SfM calculates the interior parameters simultaneously with the camera position and motion (Snavely et al., 2008; Szeliski, 2010) known as on-the-job self-calibration. Nevertheless, camera calibration in CV is not concentrating on object measurement accuracy (Aicardi et al., 2018) even the method is widely used by non-photogrammetrist due to the advantage of automation processing (Remondino et al., 2017; Jalandoni et al., 2018). Moreover, on few 3D modelling projects has found only controlled the point cloud by using the scale distance or scale bar (Jalandoni et al., 2018), which can cause some scaling issue when the actual interior parameter is unknown. This paper aims to evaluate the measurement accuracy on the 3D point cloud generated by using lightweight multispectral camera imagery with parameters derived by different calibration methods.

In carried out the experiment, the RedEdge multispectral camera was used in this study (Figure 1). The camera size is $12.1 \times 6.6 \times 4.6 \mathrm{~cm}$ dimension and 150 gram in weight, equipped with five narrowband sensors. The imaging sensor on all bands is about $4.8 \times 3.6 \mathrm{~mm}$, producing $1280 \times 960$ pixels image resolution. The default focal length provided by the manufacturer is $5.5 \mathrm{~mm}$ for all lenses. However, practically, the focal length value is different since each lens captured the image in a different wavelength. The shortest centre wavelength is on the Blue band $(475 \mathrm{~nm})$ and the maximum $840 \mathrm{~nm}$ on the Near-InfraRed channel (Table 1).

The camera is designed to use on the Unmanned Aerial Vehicle (UAV) platform for the aerial data acquisition. Thus, no viewfinder provided in the camera body. However, to use as a close-range photogrammetry tool for data acquisition, the liveview can be accessed through the camera's web-interface with the aid of a tablet or mobile device via a Wi-Fi connection. 
With the device-to-camera communication, the camera can be configured for image capture, either using the timer or snap via the virtual button. The camera supports the use of a typical battery pack as a power source, supplied through the USB cable.

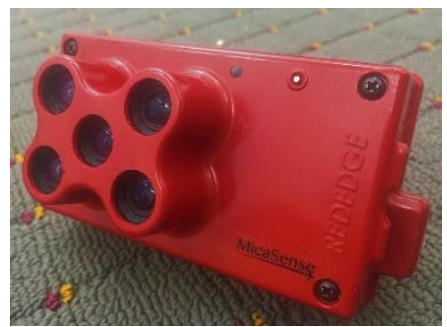

Figure 1: RedEdge multispectral camera.

\begin{tabular}{|l|c|}
\hline Sensor Size $(\mathrm{mm})$ & $4.8 \times 3.6$ \\
\hline Resolution $($ pixels $)$ & $1280 \times 960$ \\
\hline Pixel size $(\mu \mathrm{m})$ & 3.75 \\
\hline Weight $(\mathrm{g})$ & 150 \\
\hline Dimension $(\mathrm{cm})$ & $12.1 \times 6.6 \times 4.6$ \\
\hline Band & Centre Wavelength $(\mathrm{nm})$ \\
\hline Blue & 475 \\
\hline Green & 560 \\
\hline Red & 668 \\
\hline Red-edge & 717 \\
\hline Near-infrared & 840 \\
\hline
\end{tabular}

Table 1: Micasense RedEdge multispectral camera specification.

\section{METHODOLOGY}

The evaluation of the measurement accuracy on the generated point cloud was based on three different calibration method:

a) Photogrammetric self-calibration bundle-adjustment (CRP)

b) Computer vision calibration (CV)

c) On-the-job self-calibration (OTJ)

All three methods were chosen as the estimated parameters supported in the chosen point cloud processing package in this research. In this study, Agisoft Metashape was used to generate the 3D sparse point cloud and evaluates the measurement accuracy based on the respective estimated interior parameter. For the photogrammetric self-calibration bundle-adjustment approach (CRP), the parameters of the multiwavelength camera were estimated using Australis version 6. For CV calibration, the parameter was estimated using Lens, a tool to calculate the camera interior parameter provided in Metashape software. For on-the-job self-calibration (OTJ), the image was processed using Metashape with no pre-calibrated value, in other word by using uncalibrated images.

Photogrammetric calibration calculates the parameters based on the collinearity equation, while the $\mathrm{CV}$ estimates the parameter based on the projection matrix (Luhmann et al., 2016). All the calculation produces a set of parameters consisting of principal distance, principal point offset, radial distortion and decentring distortion coefficients, based on Brown (1971). The obtained parameter value calculated in the metric unit in photogrammetry, while in the CV the unit is in pixel.

\subsection{Photogrammetric self-calibration bundle-adjustment}

A $600 \times 600 \mathrm{~mm}$ test-field was used to acquire an array of 81 retro-reflective targets which placed on the vertical steel rod with different height for depth variation. The targets were having a diameter of $5 \mathrm{~mm}$ on each rod and containing a 3D coordinates used for initial value in the parameter estimation. The retro-target illuminates on the monochrome sensor of the multispectral camera with the aid of the tungsten halogen light. The light was used due to the insensitivity of the invisible band to indoor white light. Figure 2 shows the setup for photogrammetry calibration approach.

On a single exposure, the camera snaps five monochrome images simultaneously based on individual wavelength and stored into the digital memory as 12-bit TIFF format. A total of 60 images were acquired across all channel from an approximately $70 \mathrm{~mm}$ distance, in the network of mixed portrait and landscape camera orientation for the roll-diversity (Fraser, 2013).

The images then converted into JPEG as the Australis did not support the former image format. All the images processed as a multi-lenses single bundle-adjustment process (Shortis, 2012), which each image assigned according to the respective channel. The targets require manual placement on a few points before executed the automatic target detection. The software determines the centre of the reflective target with the varies weighted centroid. After all the targets were digitised, the software estimates the interior and exterior orientation parameters via free-network least square adjustment based on the known geometry of the target 3D coordinates (Robson et al., 2014). The exterior orientation then post-processed to determine the actual lens relative rotation and offset by using the following equations (Luhmann et al., 2013):

$$
\begin{aligned}
& R_{g}^{m}=R_{m}^{-1} \times R_{g} \\
& r=R_{m}\left(V_{g}-V_{m}\right)
\end{aligned}
$$

By selecting one of the lenses as the reference band, the relative rotation angle of other lenses can be calculated by multiplying the inverse rotation matrix of the master channel $\left(R_{\mathrm{m}}^{-1}\right)$ with the respective relative lenses' rotation matrix $\left(R_{g}\right)(1)$. The relative lens offset $(r)$ calculated by multiplying the rotation matrix of the master channel with the different vector between slave $\left(V_{s}\right)$ and master lenses $\left(V_{m}\right)(2)$. In this study, the Blue band was set as the reference channel.

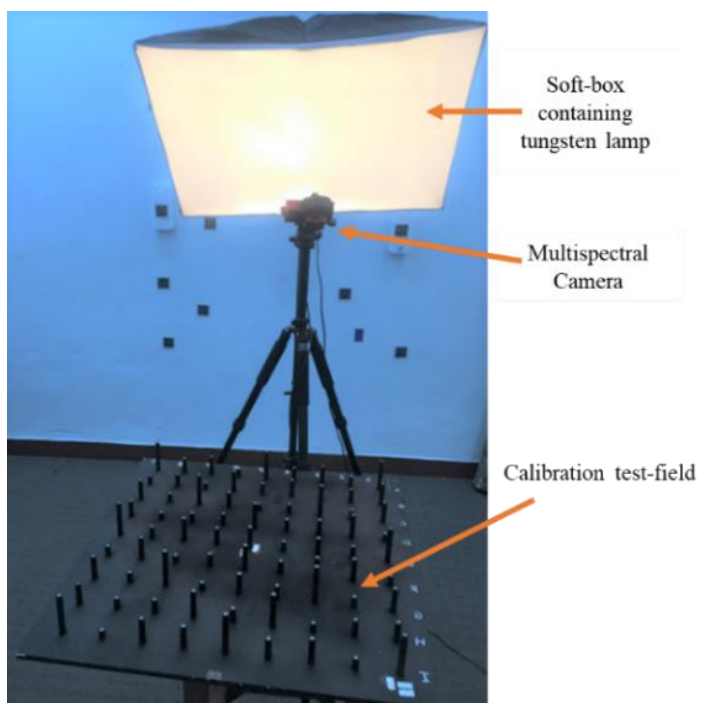

Figure 2: Calibration setup for photogrammetry approach. 


\subsection{Computer Vision Calibration}

The calibration target for parameter estimation using Lens provided by checkerboard pattern displays on the LCD screen. However, the invisible channel on the RedEdge multispectral camera (Band 4 and Band 5) were not able to detect the pattern as the screen projected the image through visible channel (RGB) formation. Thus, to enable the checkerboard image captured on all bands, the pattern was printed into A1 paper size using $50 \mathrm{~mm}$ grid size and attached into the plane surface (Figure 3). A set of 80 images across all bands from 16 camera positions with different angle acquired from an approximately $50 \mathrm{~cm}$ distance.

The images were then imported into the Lens for parameter estimation. The process is not trivial and requires no human input, as the software estimates the parameters by detecting the grid pattern automatically via image matching process and performing the bundle adjustment. The results then stored according to Metashape format (Agisoft LLC, 2019).

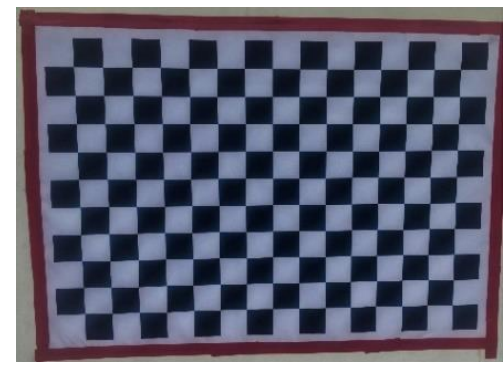

Figure 3: 2D checkerboard.

\subsection{On-the-job self-calibration}

The use of SfM/MVS photogrammetry software enables the camera interior parameters estimates simultaneously with the camera exterior position and motion without pre-calibration information during point cloud construction. As this study aims to evaluate the measurement accuracy on the $3 \mathrm{D}$ point cloud generated using parameters from different estimation method, the on-the-job self-calibration requires no initial camera's orientation information to be input for the processing.

The only required initial value is the focal length, gained from the image's EXIF information, and the algorithm calculates the interior parameters as well as the rotation angle and lens offset automatically based on image matching procedure. While for the estimated value using CRP and CV, the parameters were entered into the camera calibration information in Metashape. All the parameters then fixed to ensure the software not adjusting the pre-calibrated value. Regarding CRP calibration, the estimated interior parameter was converted from millimetre to pixel unit by using a built-in conversion tool in Metashape. For Lens estimated value, the calibrated output was already stored in Metashape format, and only recalled the pre-calibrated information into the project setup.

The measurement accuracy assessment was conducted on three different verification check-fields comprising different object size, texture, and depth variation. The Check-Field_1 (Figure 4) contained an array of check-targets, attached on the plane surface with a dimension of 4 x $1 \mathrm{~m}$. The Check_Field_2 (Figure 5) is a $10 \times 4 \mathrm{~m}$ plane wall containing a colour-rich mural drawing, and the targets located at the edge of the mural. The Check_Field_3 (Figure 6) is an 11 x 5 m building wall containing different depth variation. The $\mathrm{X}$-axis is running along the check-field surface, $\mathrm{Y}$-axis is perpendicular to the check-field (depth), and the Z-axis is along the vertical direction. Table 2 summarises the number of acquired images, control points, check-points, and check-distance used for accuracy verification.

\begin{tabular}{|l|c|c|c|c|}
\hline Check-Field & $\begin{array}{c}\text { Number } \\
\text { of } \\
\text { Images }\end{array}$ & $\begin{array}{c}\text { Number } \\
\text { of Control } \\
\text { Points }\end{array}$ & $\begin{array}{c}\text { Number } \\
\text { of Check } \\
\text { Points }\end{array}$ & $\begin{array}{c}\text { Number } \\
\text { of Check } \\
\text { Distances }\end{array}$ \\
\hline Check_Field_1 & 150 & 5 & 34 & 14 \\
\hline Check_Field_2 & 270 & 5 & 8 & 8 \\
\hline Check_Field_3 & 230 & 6 & 34 & 17 \\
\hline
\end{tabular}

Table 2: Number if images acquired, control and checkpoints, and check distances.

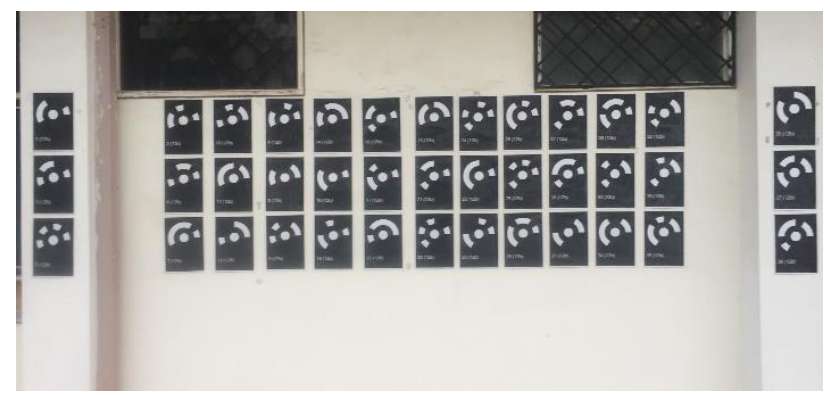

Figure 4: Check_Field_1

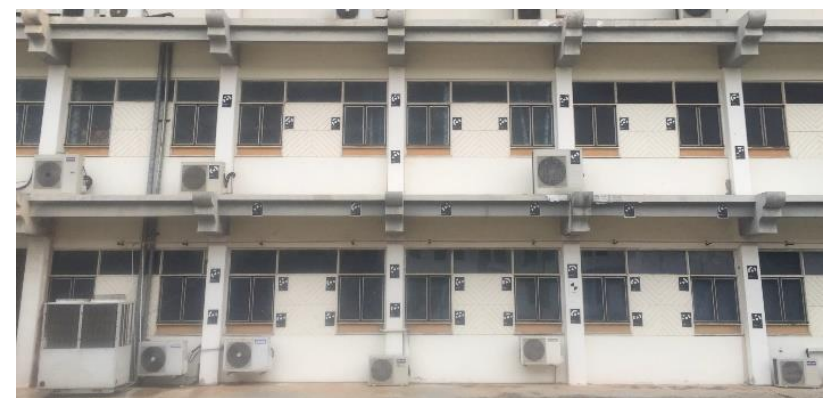

Figure 5: Check_Field_2

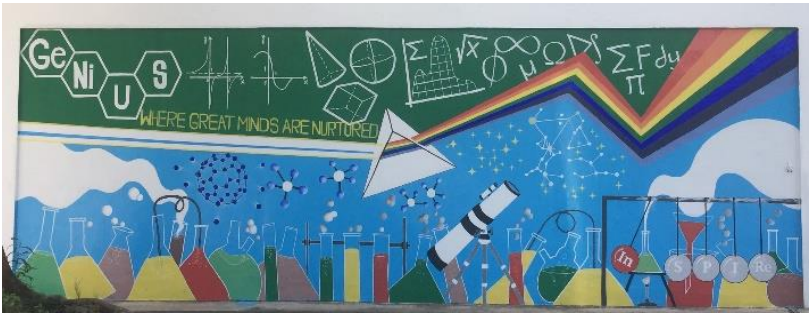

Figure 6: Check_Field_3

Generally, the processing sequence was similar for all datasets, starting with the project setup, and followed by the point cloud construction through the image alignment process. Then, the targets on the point cloud were marked, used for control as well as for verification purpose. The initially generated point cloud then re-optimised to determine the final point coordinate through the bundle-adjustment process after the cleaning process. For measurement analysis, the calculated coordinate on check-points and length on the check-distance were extracted.

The coordinate system was set to local for arbitrary 3D model construction. For the measurement accuracy, the marker and scale bar precision were set to $0.001 \mathrm{~m}$, as the standard deviation of the target's coordinate measured using total station was less than $1 \mathrm{~mm}$. On the image coordinate accuracy setup, 
the precision of the marker can be set as tight as 0.1 pixels for automatic target detections, to up to 1 pixel for manual marking. On this experiment, the value was set to 0.1 pixels for Check_Field_1 and Check_Field_2 dataset, and 0.5 pixels for Check_Field_3 project due to a few of the points were manually marked.

The point cloud generation commenced with the image alignment process. The parameter for image alignment processing was followed USGS (2017) guideline for an unlimited number of key-point and tie-point detection and construction. Then the placement of the target on the constructed point cloud was performed automatically, by gradually setting the tolerance weight from 50 to 100 until all the targets successfully marked. For the target which not automatically detect, then a manual input was implemented as it applied on Check_Field_3 dataset.

Each control and verification point contained 3D coordinate determined by using Trimble M3 reflector-less Total Station based on intersection method. Check-Field_1 and Check_Field_2 were observed from two stations intersection, while the Check_Field_3 coordinates were measured from three-station setup. All the coordinates from respective checkfield were undergone least-square adjustment using StarNET software to determine the precision of the observed reference coordinates. The adjustment yielded the precision of less than one $\mathrm{mm}$ for all three check-fields.

The generated point cloud then underwent a cleaning process by using the 'Gradual Selection' tool to remove the noise due to the wrong projection during the image matching process. The filtering processing step can be found on USGS (2017) and Mayer and Kersten (2018). This step is necessary to remove the miss-projected points based on calculated variance to obtained a final corrected point cloud. After each cleaning steps, the point cloud re-optimised to calculate the new point coordinates.

In this study, the point cloud accuracies were evaluated based on check-point and check-distance error. For the check-point error analysis, the coordinate estimated by Metashape using the respective calibrated parameter was compared to the coordinate measured with the Total Station. Similarly, for distance error, the comparison was performed based on extracted distance on the point cloud and compared with distance measured from Total Station. However, for distance error analysis, the generated point cloud constrained by a single scale-distance, which the 'true' distance value obtained from the Total Station observation. The accuracy was determined by using the root mean square error, RMSE, where the comp refers to the coordinates extracted from the 3D model and ref indicates the coordinate of the same point derived using Total Station (Remondino et al., 2017):

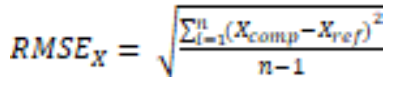

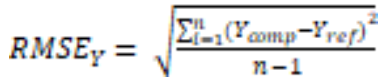

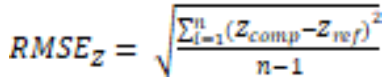

$$
\begin{aligned}
& R_{M S E_{X Z Y}}=\sqrt{R M S E_{X}^{2}+R M S E_{Z}^{2}+R M S E_{Y}^{2}}
\end{aligned}
$$

\section{RESULT AND DISCUSSION}

Table 3 summarises the parameters obtained from the CRP calibration method comprising calibrated focal length (principal distance) in $\mathrm{mm}$ and pixel value (after conversion in Metashape), lens relative rotation in omega, phi and kappa ( $\omega$, $\varphi, \kappa)$ and lens relative offsets $(X, Y, Z)$ calculated based from Blue channel. The calibrated value remains fixed on all check field datasets. Note that only principal distance shows in the table, however, all calibrated interior parameters were used in Metashape.

Table 4 shows the pre-calibrated value using the CV method and adjusted value based on the SfM algorithm using Metashape processing. The CV calibration only determines the interior parameters (only focal length show on the table) and the slave lens offset and rotation angle were estimated based on the $\mathrm{SfM}$ algorithm in Metashape. The value also remains fixed on all check filed datasets. In Table 4 also shown that the value of focal length estimated by OTJ were varies on different checkfields, as it were the usual effect since the algorithm estimates the interior value based on the image scene-dependent and the number of control point used.

Table 5 reports the measurement RMSEs based on different verification check-field for error analysis. From the result, it shows that using the parameter obtained from photogrammetric self-calibration bundle-adjustment (CRP) provides a small error on all experiments. On the first two check-fields, which consisted of flat surfaces show a similar accuracy on point cloud generated using automatic on-the-job self-calibration (OTJ) and $\mathrm{CV}$ pre-calibration parameters. However, on the surface with depth variation check-field (Check_Field_3) shows the point cloud generated by using OTJ and CRP produced a similar measurement accuracy. It indicates that the automatic calibration approaches performing well on the surface with different depth. Figure 7 to Figure 9 show the individual error $\left(\mathrm{RMSE}_{\mathrm{XZY}}\right)$ compared with the true value, where the CRP consistently shows small errors on all datasets.

\begin{tabular}{|c|c|c|c|c|c|c|c|c|}
\hline \multirow{2}{*}{ Lens } & \multicolumn{2}{|c|}{ Principal Distance } & \multicolumn{3}{|c|}{ Lens Relative Rotation ( $\left.{ }^{\circ}\right)$} & \multicolumn{3}{|c|}{ Lens Relative Offset (mm) } \\
\hline & $(\mathbf{m m})$ & (pixel) & $\omega$ & $\varphi$ & $\kappa$ & $\mathbf{X}$ & $\mathbf{Y}$ & $\mathbf{Z}$ \\
\hline Blue & 5.438 & 1449.674 & \multicolumn{6}{|c|}{ Reference channel } \\
\hline Green & 5.422 & 1445.964 & -0.003 & -0.008 & 0.272 & -30.659 & -0.013 & -0.696 \\
\hline Red & 5.417 & 1444.319 & -0.004 & -0.009 & 0.253 & -30.116 & -21.627 & -0.207 \\
\hline Rededge & 5.432 & 1448.424 & 0.002 & -0.006 & 0.078 & -15.013 & -10.810 & -0.177 \\
\hline NIR & 5.453 & 1454.069 & -0.003 & -0.003 & -0.087 & -0.003 & -21.845 & -0.545 \\
\hline
\end{tabular}

Table 3: Calibrated parameters obtained using the CRP method. 
The International Archives of the Photogrammetry, Remote Sensing and Spatial Information Sciences, Volume XLII-4/W16, 2019 6th International Conference on Geomatics and Geospatial Technology (GGT 2019), 1-3 October 2019, Kuala Lumpur, Malaysia

\begin{tabular}{|l|c|c|c|c|}
\hline Focal Length & \multirow{2}{*}{ CV (pixel) } & \multicolumn{3}{|c|}{ OTJ (pixel) } \\
\cline { 3 - 5 } Lens & & Check_Field_1 & Check_Field_2 & Check_Field_3 \\
\hline Blue & 1453.029 & 1462.449 & 1449.385 & 1453.277 \\
\hline Green & 1449.319 & 1464.226 & 1448.346 & 1445.902 \\
\hline Red & 1445.553 & 1458.460 & 1448.420 & 1445.901 \\
\hline Rededge & 1450.408 & 1455.670 & 1450.724 & 1450.481 \\
\hline NIR & 1456.565 & 1466.425 & 1458.071 & 1457.544 \\
\hline
\end{tabular}

Table 4: Calibrated focal length using the CV method and OTJ automatic calibration.

\begin{tabular}{|c|c|c|c|c|c|c|c|c|c|}
\hline & \multicolumn{3}{|c|}{ ОTJ } & \multicolumn{3}{|c|}{$\mathrm{CV}$} & \multicolumn{3}{|c|}{ CRP } \\
\hline & $\begin{array}{c}\mathrm{RMSE}_{\mathrm{XZ}} \\
(\mathrm{mm})\end{array}$ & $\begin{array}{l}\text { RMSE }_{Y} \\
(\mathrm{~mm})\end{array}$ & $\begin{array}{l}\text { RMSE }_{X Z Y} \\
(\mathrm{~mm})\end{array}$ & $\begin{array}{c}\text { RMSE }_{\mathrm{XZ}} \\
(\mathrm{mm})\end{array}$ & $\begin{array}{c}\mathrm{RMSE}_{Y} \\
(\mathrm{~mm})\end{array}$ & $\begin{array}{l}\text { RMSE }_{X Z Y} \\
(\mathrm{~mm})\end{array}$ & $\begin{array}{c}\text { RMSE }_{\text {XZ }} \\
(\mathrm{mm})\end{array}$ & $\begin{array}{c}\mathrm{RMSE}_{Y} \\
(\mathrm{~mm})\end{array}$ & $\begin{array}{c}\text { RMSE }_{X Z Y} \\
(\mathrm{~mm})\end{array}$ \\
\hline Check_Field_1 & 4.74 & 2.50 & 5.36 & 1.61 & 4.80 & 5.06 & 0.44 & 1.05 & 1.14 \\
\hline Check_Field_2 & 1.31 & 2.83 & 3.12 & 1.74 & 3.29 & 3.72 & 1.24 & 2.11 & 2.45 \\
\hline Check_Field_3 & 2.59 & 1.92 & 3.23 & 3.23 & 4.42 & 5.47 & 2.32 & 2.48 & 3.40 \\
\hline
\end{tabular}

Table 5: RMSE on check-point measurement

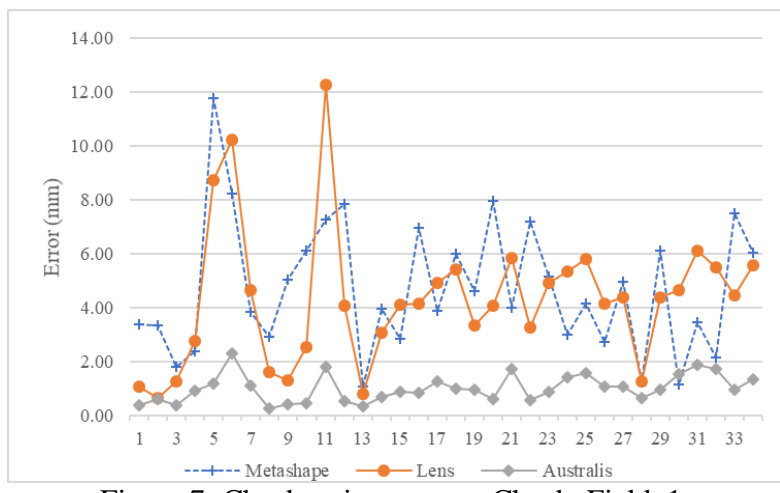

Figure 7: Check-point error on Check_Field_1.

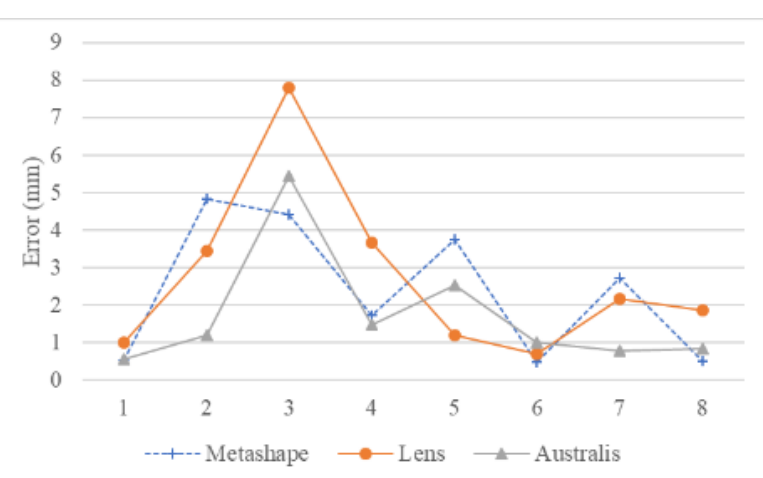

Figure 8: Check-point error on Check_Field_2.

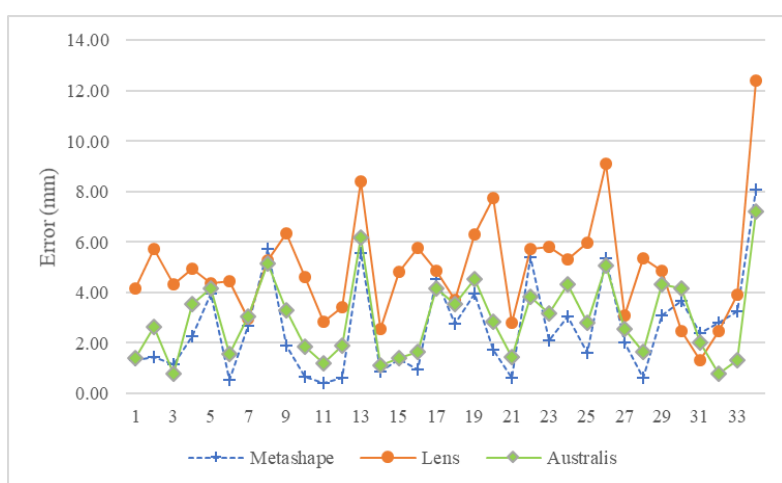

Figure 9: Check-point error on Check_Field_3.

On more in-depth analysis using ANOVA, the reported RMSE $_{\text {XZY }}$ on Check_Field_2 shows a similar accuracy on all estimated parameter. While for Check_Field_1 and Check-
Field_3 report a significant difference. Further post hoc test analysis using two-tail t-test confirmed that the parameter estimated using OTJ and CV calibration were significantly different with CRP on Check_Field_1, while on Check_Field_3 only the CV parameter not significant. It can be concluded that using both automatic OTJ and CRP calibration provided a similar outcome when the point cloud constrained with the control points.

Table 6 to Table 8 reports the error on distance measurement on all check-fields. This experiment constrained the bundleadjustment calculation using only a single scale-distance. This experiment executed to analyse the error on point cloud when no control point provided in the 3D point cloud processing. Based on the result, the parameter estimated from the CRP method produced the most accurate results on all check-fields. Contrary, the automatic OTJ method produced the worst 3D model on all three different check-field, indicates the inconsistency of $3 \mathrm{D}$ point cloud construction using with and without control points.

The numerical results can be supported on the individual distance error shown by Figure 10 to Figure 12. Based on the ANOVA statistical test, the variance on all parameters was significantly different. Using post hoc t-test analysis, the distance error using Australis was significantly different on all check-field, shows the reliability of using photogrammetry selfcalibration bundle adjustment method to generate an accurate 3D model for the close-range object, either weighted by control point or distance.

\begin{tabular}{lccc}
\hline & OTJ & CV & CRP \\
\hline Mean $(\mathbf{m m})$ & 3.02 & 2.96 & 0.61 \\
\hline Min $(\mathbf{m m})$ & 0.46 & 0.61 & 0.00 \\
\hline Max $(\mathbf{m m})$ & 6.35 & 8.73 & 3.51 \\
\hline RMSE $(\mathbf{m m})$ & 3.83 & 3.79 & 1.11 \\
\hline
\end{tabular}

Table 6: Distance error on Check_Field_1.

\begin{tabular}{cccc}
\hline & OTJ & CV & CRP \\
\hline Mean (mm) & 3.02 & 3.21 & 0.97 \\
\hline Min (mm) & 0.23 & 0.01 & 0.05 \\
\hline Max (mm) & 7.74 & 6.55 & 2.66 \\
\hline RMSE (mm) & 5.34 & 4.13 & 1.36 \\
\hline
\end{tabular}

Table 7: Distance error on Check_Field_2. 


\begin{tabular}{cccc}
\hline & OTJ & CV & CRP \\
\hline Mean $(\mathbf{m m})$ & 5.50 & 4.02 & 2.04 \\
\hline Min $(\mathbf{m m})$ & 0.55 & 1.08 & 0.27 \\
\hline Max $(\mathbf{m m})$ & 10.77 & 9.30 & 5.11 \\
\hline RMSE $(\mathbf{m m})$ & 6.76 & 4.85 & 2.54 \\
\hline
\end{tabular}

Table 8: Distance error on Check_Field_3.

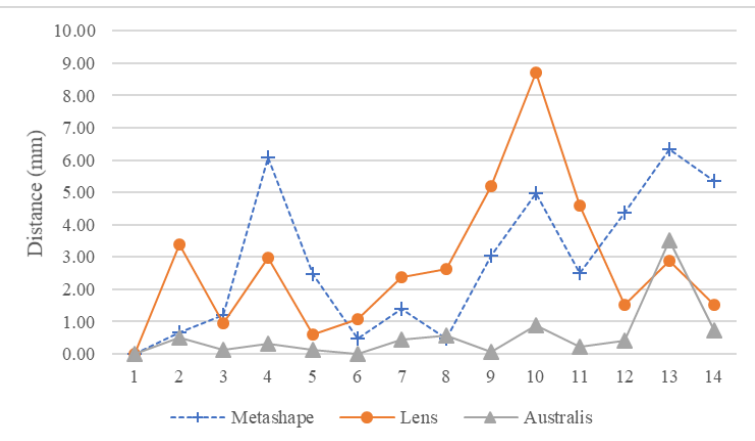

Figure 10: Check-distance error on Check_Field_1.

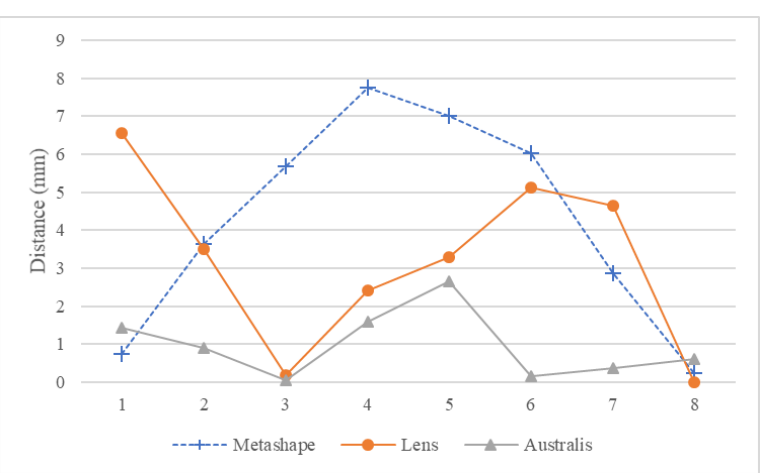

Figure 11: Check-distance error on Check_Field_2.

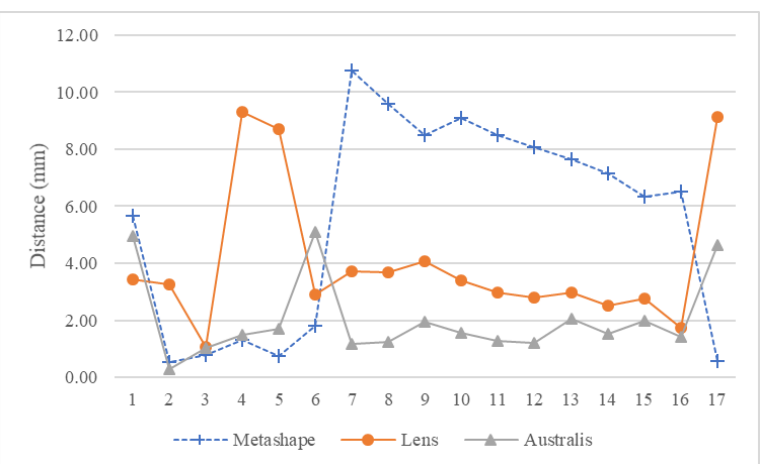

Figure 12: Check-distance error on Check_Field_3.

\section{CONCLUSION}

This paper reports the measurement accuracy on 3D point cloud generated using a multispectral camera and processed by SfM/MVS photogrammetry for close-range application. The result from this study provides a reference on our main research, which using the multispectral camera for close-range 3D modelling of heritage object documentation. As SfM/MVS photogrammetric processing provides an automatic approach to generate the 3D model using uncalibrated images, the result from this study shows the inconsistency on the point cloud accuracy when no control point used. In other words, using only the scale bar yielded inaccurate 3D model without knowing the actual interior parameter.
The result produced by OTJ is accurate when the object has a different depth variation. Therefore, it is necessary to provide the control points during data acquisition for automatic calibration and producing accurate 3D modelling by using SfM/MVS. In the other hand, using the actual interior and relative orientation parameter derived using CRP calibration approach produced a consistent result on both verification test at all three surfaces. Nevertheless, estimating the interior parameter using the CRP might be trivial for nonphotogrammetrist, requires test-field setup and post-processing on exterior orientation parameters to calculate the relative rotation and offset of all lenses.

This study executed by using a typical parallel camera configuration for data acquisition and 3D modelling. Hence, it is necessary to investigate the measurement accuracy when the convergent and oblique camera with roll diversity included in the processing.

The test on this experiment only adopted check-point and check-distance for verification, probably produced a biased result especially on Check_Filed_2 due to a limited number of verification points. Therefore, for future work, the point cloud generated using SfM/MVS photogrammetry will compare with the point cloud obtained from terrestrial laser scanning data, allowing all points on the entire model use for analysis. Moreover, the effect of the number of control points towards accuracy also will be investigate

\section{ACKNOWLEDGEMENTS}

The corresponding author highly acknowledged Universiti Teknologi MARA and Malaysian Higher Education Ministry for the financial aid under the SLAB scheme. Also, the corresponding author acknowledged Universiti Teknologi Malaysia for the research activities under Vote 05G12 and RUG Tier 2 Vote No. 15J12.

\section{REFERENCES}

Agisoft LLC (2019). 'Agisoft Metashape User Manual'. Available at: https://www.agisoft.com/pdf/metashapepro_1_5_en.pdf.

Aicardi, I., Chiabrando, F., Maria Lingua, A. and Noardo, F. (2018). 'Recent Trends in Cultural Heritage 3D Survey: The Photogrammetric Computer Vision Approach', Journal of Cultural Heritage.

Brown, D. C. (1971). 'Close-Range Camera Calibration', Photogrammetric Engineering, pp. 855-866.

Fraser, C. S. (2013). 'Automatic Camera Calibration in Close Range Photogrammetry', Photogrammetric Engineering \& Remote Sensing, 79(4), pp. 381-388.

Jalandoni, A., Domingo, I. and Taçon, P. S. C. (2018). 'Testing the Value of Low-Cost Structure-from-Motion (SfM) Photogrammetry for Metric and Visual Analysis of Rock Art', Journal of Archaeological Science: Reports. Elsevier, 17(December), pp. 605-616.

Liang, H., Lucian, A., Lange, R., Cheung, C., Su, B., Shing, C., Su, B., Cheung, C. and Su, B. (2014). 'Remote Spectral Imaging with Simultaneous Extraction of 3D Topography for Historical 
Wall Paintings', ISPRS Journal of Photogrammetry and Remote Sensing, 95, pp. 13-22.

Luhmann, T., Fraser, C. S. and Maas, H. G. (2016). 'Sensor Modelling and Camera Calibration for Close-Range Photogrammetry', ISPRS Journal of Photogrammetry and Remote Sensing. International Society for Photogrammetry and Remote Sensing, Inc. (ISPRS), 115, pp. 37-46.

Luhmann, T., Robson, S., Kyle, S. and Boehm, J. (2013). Closerange photogrammetry and $3 D$ imaging. Walter de Gruyter.

Mayer, C. and Kersten, T. P. (2018). 'A Comprehensive Workflow to Process UAV Images for the Efficient Production of Accurate Geo-Information A Comprehensive Workflow to Process UAV Images for the Efficient Production of Accurate Geo-Information', IX Conferencia Nacional de Cartografia e Geodesia, (November), pp. 1-8.

Remondino, F. and Fraser, C. S. (2006). 'Digital Camera Calibration Methods: Considerations and Comparisons', International archives of photogrammetry and remote sensing, 36(5), pp. 266-272.

Remondino, F., Nocerino, E., Toschi, I. and Menna, F. (2017). 'A Critical Review of Automated Photogrammetric Processing of Large Datasets', ISPRS - International Archives of the Photogrammetry, Remote Sensing and Spatial Information Sciences, XLII-2/W5(September), pp. 591-599.

Robson, S., MacDonald, L., Kyle, S. and Shortis, M. (2014). 'Multispectral Calibration to Enhance the Metrology Performance of C-Mount Camera Systems', International Archives of the Photogrammetry, Remote Sensing and Spatial Information Sciences - ISPRS Archives, 40(5), pp. 517-521.

Shortis, M. R. (2012). 'Multi-Lens, Multi-Camera Calibration of Sony Alpha NEX 5 Digital Cameras', CEUR Workshop Proceedings, 1328(December).

Snavely, N., Seitz, S. M. and Szeliski, R. (2008). 'Modeling the World from Internet Photo Collections', International Journal of Computer Vision, 80(2), pp. 189-210.

Szeliski, R. (2010). 'Computer Vision: Algorithms and Applications', Computer, 5, p. 832.

Torres-Martínez, J. A., Sánchez-Aparicio, L. J., HernándezLópez, D. and González-Aguilera, D. (2017). 'Combining Geometrical and Radiometrical Features in the Evaluation of Rock Art Paintings', Digital Applications in Archaeology and Cultural Heritage. Elsevier Ltd, 5(March), pp. 10-20.

Tsai, R. Y. (1986). 'An Efficient and Accurate Camera Calibration Technique for 3D Machine Vision', Proc. of Comp. Vis. Patt. Recog., pp. 364-374.

USGS (2017). 'Unmanned Aircraft Systems Data PostProcessing', USGS National UAS Project Office, (1), pp. 1-18. Available at: https://www.faa.gov/uas/. 